\title{
Filament activity and photospheric magnetic evolution related to flares
}

\author{
Y. Jiang ${ }^{1,2}$ and J. Wang ${ }^{1}$ \\ 1 Beijing Astronomical Observatory, National Astronomical Observatories, Chinese Academy of Science, \\ Beijing 100012, PR China \\ 2 Yunnan Astronomical Observatory, Chinese Academy of Science, Kunming 650011, PR China \\ e-mail: jych@ourstar.bao.ac.cn
}

Received 20 March 2000 / Accepted 25 August 2000

\begin{abstract}
Three successive activations of an active-region filament were observed over a period of eight hours. Each disturbance showed distinctive characteristics and was followed by a flare of different properties. The filament had one end rooted in strong $\delta$-sunspots. During the first activation, the filament rose up at the rooting end and detached from the $\delta$-sunspots while its main body remained in place and separated into two twisted threads. During the second activation, only one thread was disturbed and finally disappeared; twisted threads, however, appeared during the third activation. The evolution of photospheric magnetic fields, associated with the filament disturbances, was characterized by squeezing and shearing of $\delta$-sunspots, flux cancellation and emergence beneath the two ends of the filaments. Our data suggests that magnetic reconnection in the photospheric layer is the likely cause of filament destabilisation.
\end{abstract}

Key words. Sun: prominences - Sun: filaments - Sun: activity - Sun: flares - Sun: magnetic fields

\section{Introduction}

Filaments, known as absorption features on the solar disk in the $\mathrm{H}_{\alpha}$ line, always form above the polarity inversion zone of photospheric magnetic fields. Their disturbances are closely related to flares and coronal mass ejections (Tandberg-Hanssen 1995). The flare-associated filament disturbances often take place before the onset of flares. They exhibit varied behaviors, such as breaking into fragments, ascending from one end, and partially or wholly erupting (Martin 1980; Tang 1986; McAllister et al. 1996; Mouradian et al. 1998).

In their quiescent phase, filaments always have an average upward motion (Schmieder 1990; Wang et al. 1996a). In the activating phase, however, they often show a complicated Doppler pattern. Twisted structures and motions are frequently observed, suggesting the existence of twisted magnetic fields in a disturbed filament (Schmieder et al. 1985; Kurokawa et al. 1987; Raadu et al. 1988; Vísnak et al. 1991; Rompolt 1990; Wang et al. 1996a; Jiang \& Wang 2000).

The magnetic field in filaments plays an important role in their formation and destabilisation. Generally, the loss of filament equilibrium and associated flare activity are

Send offprint requests to: Y. Jiang,

e-mail: jyc@cosmos.ynao.ac.cn related to the reconstruction of the magnetic field in and around the filament. Some observations suggest that the local photospheric field evolution, such as motions of pores and sunspots and emergence and cancellation of magnetic flux, may trigger destabilisations of filaments; in some cases, the disturbances may be associated with changes in adjacent, large-scale magnetic structures (Rust et al. 1975; Simon et al. 1986; Hermans \& Martin 1986; Gaizauskas 1989; Schmieder 1990; Martin \& Livi 1992; Feynman \& Martin 1995; Wang et al. 1996a; Jiang \& Wang 2000).

An understanding of the physical process of filament disturbance is important in the study of filament structure and related phenomena, such as flare and CME. The detailed observations of the morphology, evolution and dynamic processes in disturbed filaments and the careful studies of the changes in neighboring fields during the course of filament disturbance may provide clues to understand filament disturbance.

In this paper, we study the disturbances of a filament and associated flares in AR6619 on May 10, 1991. The observations were made by the Solar Magnetic Field Telescope (SMFT), installed at Huairou Solar Observing Station (HSOS) of the Beijing Astronomical Observatory. The filament disturbances had some remarkable features: the filament was divided into two twisted threads after the first disturbance; in the following disturbances, each 


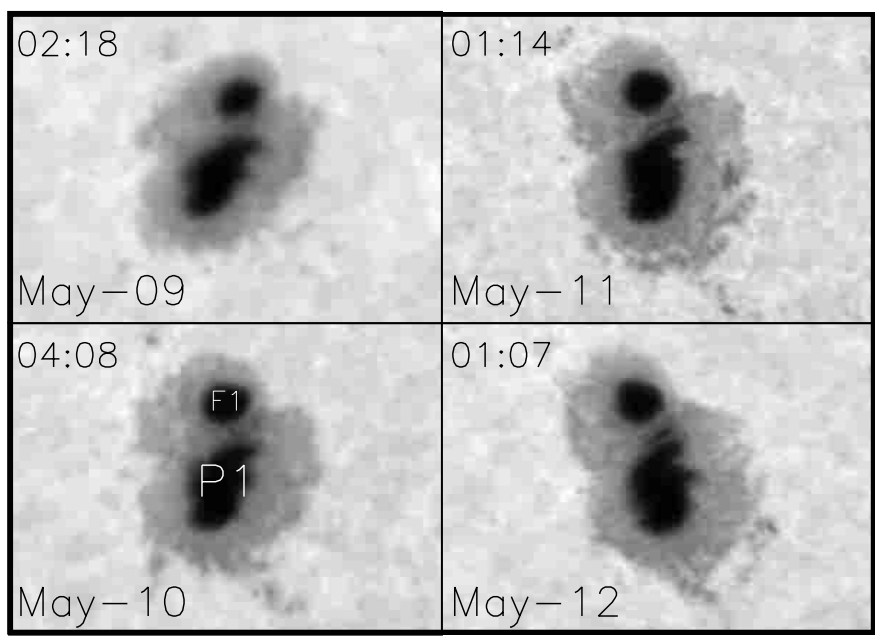

Fig. 1. Time sequences of FeI5324 ̊/0.150 ̊ filtergrams from May 9 to 12 for AR6619. The preceding and following sunspots are denoted as P1 and F1. Top is North and West is to the right. The field of view (FOV) is $205^{\prime \prime} \times 150^{\prime \prime}$

thread of the filament showed different behaviors; each disturbance preceded a flare. We have obtained complete sets of $\mathrm{H}_{\beta}$ Dopplergrams and filtergrams, which enable an accurate description of the evolution of filament threads. The relationship among filament disturbances, flares and changes in the photospheric magnetic field has been studied in detail. The next section is a brief description of the instrumentation and observation. In Sect. 3, flares associated with filament disturbances are described. In Sect. 4, the dynamics of the filament and the evolution of photospheric magnetic fields are detailed. The final section is our conclusion and discussion.

\section{Observation}

\subsection{Instrument}

The SMFT, a vector videomagnetograph system, records the chromospheric $\mathrm{H}_{\beta}$ line with a $0.125 \AA$ bandpass and the photospheric Fe $5324 \AA$ line with a $0.150 \AA$ bandpass (Ai \& Hu 1986). In the present study, the data base includes narrow band filtergrams, line-of-sight and transverse magnetograms in the photosphere, $\mathrm{H}_{\beta}$ filtergrams and Dopplergrams in the chromosphere. The seeing was fairly good during the eight-hour observation. The spatial resolution was $2-3$ arcsec for both magnetograms and Dopplergrams, and the temporal resolution was several minutes for the Dopplergrams during flare and less than one hour for the line-of-sight magnetograms of the photosphere. Unfortunately, only a few transverse magnetograms were taken.

Calibration of HSOS vector magnetograms in the photosphere is made based on the following relations:

$B_{\|}=C_{\|} \frac{V}{I}$

$B_{\perp}=C_{\perp}\left(\frac{Q^{2}}{I^{2}}+\frac{U^{2}}{I^{2}}\right)^{1 / 4}$ where $C_{\|}$and $C_{\perp}$ are the calibration coefficients for the line-of-sight and transverse magnetograms, respectively; $I, V, Q$ and $U$ are four narrow-band images of Stokes parameters. The noise level is less than $20 \mathrm{G}$ for line-of-sight magnetograms, while for transverse field measurement, it is less than $150 \mathrm{G}$ (see Wang et al. 1996b).

The $\mathrm{H}_{\beta}$ Dopplergram calibration is based on the relation

$V_{\|}=C_{\|} \frac{I_{\mathrm{b}}-I_{\mathrm{r}}}{I_{\mathrm{b}}+I_{\mathrm{r}}}$,

where $C_{\|}$is the calibration parameter obtained from solar rotation observation, $I_{\mathrm{r}}$ and $I_{\mathrm{b}}$ are the intensity of the red and blue wing ( $\pm 0.24 \AA$ from line center). The processing and limitation of HSOS filter-based Dopplergrams were described and discussed by Wang (1993) and Wang et al. (1996a).

\subsection{General evolution of the active region and filaments}

The active region NOAA6619 (N32, L264) was a compact $\delta$ configuration group with the central meridian passage on May 11, 1991. Its appearance on four successive days, May 9-12, is shown in Fig. 1. The region had the following remarkable features: (a) it consisted of two quasi-regular sunspots P1 and F1 with opposite polarity, which locked together in a common penumbra and never separated during the passage of the region across the solar disk; (b) it showed reversal polarity as compared to the Hale-Nichols polarity law; (c) the axial direction of the sunspot group, from the following spot F1 to the proceeding spot P1, had a counterclockwise rotation. On May 10, the axis of the sunspot group was nearly perpendicular to the sun's equator. It rotated about $6^{\circ}$ in an interval about 8 hours.

The evolution of the filament and corresponding magnetic field in the photosphere from May 9 to 11 is shown in Fig. 2. The HSOS $\mathrm{H}_{\beta}$ observations showed that the filament stayed visible during the AR's passage across the solar disk. On May 9, the filament was continuously activated during our observation from 01:04 to 02:11 UT; the black arrow in the Dopplergram indicated a pair of opposite Dopplershifts along its main axis. On May 10, the filament underwent three obvious disturbances, which were each accompanied by three flares. In this paper, we focus on these three events, and refer to them as the first, second and third filament activation (and flare). The $\mathrm{H}_{\beta}$ filtergram of Fig. $2 \mathrm{~b}$ shows the appearance of the filament near the maximum of the first flare. We see that the filament consisted of two threads, Thd1 and Thd2. They intersected at two points, $\mathrm{A}$ and $\mathrm{B}$, then extended to the southwestern end (marked as SW), the section, BC, and the northeastern end (marked as NE). On May 11, two threads of the filament (indicated by two small arrows) were seen, but they did not exhibit obvious changes in either $\mathrm{H}_{\beta}$ filtergrams or Dopplergrams during observation.

In magnetograms of Fig. 2, the line-of-sight magnetic field underneath the central part of the filament was rather 


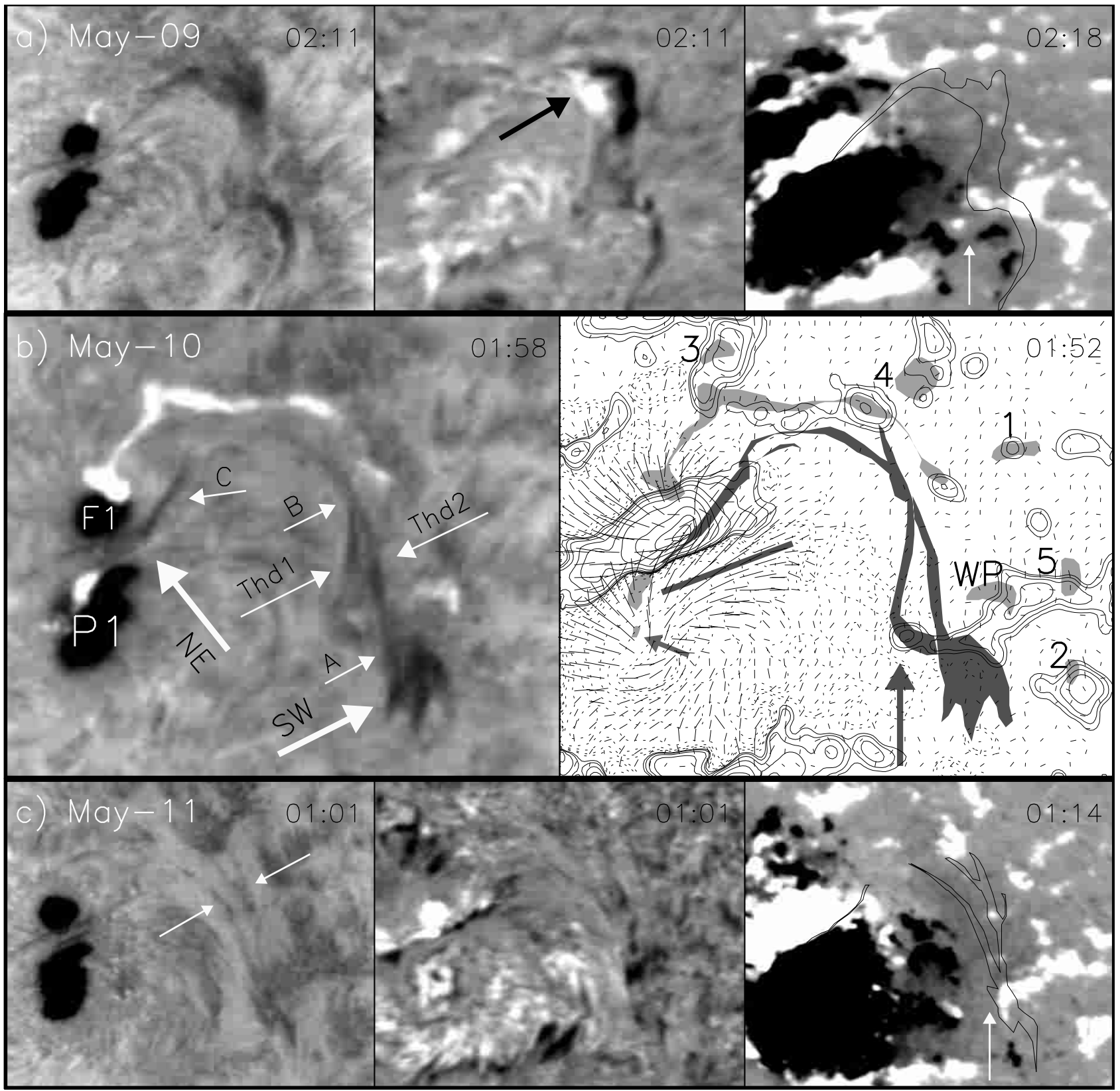

Fig. 2. a) and c) The offband (-0.24 $\AA) \mathrm{H}_{\beta} / 0.125 \AA$ filtergrams (left), $\mathrm{H}_{\beta}$ Dopplergrams (middle) and line-of-sight magnetograms (right) with filament superposed as dark contour on May 9 and 11; the black (white) patches in Dopplergrams correspond to blueshifts (redshifts) and the brighter (darker) patches in magnetograms represent positive (negative) polarities. b) The offband $\mathrm{H}_{\beta}$ filtergram (left) at 01:58 UT and the vector magnetogram (right) at 01:52 UT superposed by the filament as dark ribbons and flares as grey ribbons and patches on May 10. The flaring patches of the second flare are also overlapped by grey patches and numbered as "1", "2", "3", "4",and "5" (see the description in Sect. 3); the short arrow indicates a bright patch appearing in the umbra of the P1. The line-of-sight field is shown by isogauss solid (dashed) contours for positive (negative) polarity with levels $\pm 20,40,80,160,320,640,1280,1920,2560$ and $2880 \mathrm{G}$, the transverse field is presented by short line segments with length proportional to the field strength and alignment parallel to the field direction. The FOV is $215^{\prime \prime} \times 180^{\prime \prime}$

weak; but near its two ends, we observed the following points. (a) The NE end terminated between opposite polarities of the $\delta$ spot. As stated by Zirin \& Liggett (1987), this is a condition in favor of flares. (b) Near the SW end, magnetic flux was seen obviously cancelling on May 10. As indicated by the long vertical arrow, flux cancellation was probably caused by the intruding of positive flux into the negative flux of the P1. Such intrusion, as indicated by vertical arrows, started on May 9 and stopped on May 11. Taking the filament behavior into account, it seems that such intrusion and the resulting flux cancellation played an important role in destabilising the filament; this will be discussed further in Sect. 4 . 
Table 1. Filament activations and flares on May 10, 1991

\begin{tabular}{l|l|c}
\hline NO. & filament activations & flares \\
\hline 1 & $00: 16-01: 24$ UT & $\begin{array}{c}01: 15-01: 55-03: 06 ~ U T \\
\text { (start) (peak) (end) }\end{array}$ \\
\hline 2 & $01: 54-03: 06$ UT & $02: 25-04: 18$ UT \\
\hline 3 & $03: 46-07: 43$ UT & $06: 09,06: 59$ UT \\
\hline
\end{tabular}

\section{Flares associated with filament disturbances on May 10, 1991}

The first flare of $\mathrm{H}_{\alpha}$ importance $1 \mathrm{~F}$ and X-ray class $\mathrm{C} 5.8$ occurred at N34E18 with start, peak and end times around 01:15 UT, 01:55 UT and 03:06 UT, respectively (according to Solar-Geophysical Data, No. 567, Part II, 1991). It consisted of two obvious ribbons (Fig. 2b): a small, southern ribbon over the umbra of the P1 and a large, northern one almost parallel to the filament axis. They appeared in regions of opposite polarity and showed no measurable increase in separation and ultimately faded away in place. An exception was the bright patch, "WP", which continuously increased in size from 01:54 to 02:33 UT, then faded away. After the maximum phase of the first flare, the second flare is shown by the time sequences of $\mathrm{H}_{\beta}$ filtergrams in Fig. 3b. The new bright patches, "1" at 02:25 UT, "2" and " 3 " at 02:33 UT, as well as "4" and "5" at 02:57, appeared on the north of the filament; they were not the result of spreading and expansion of the previous flare ribbons. They formed the northern ribbon which moved away from the filament and faded one hour later. The southern ribbon was also observed in the umbra of the P1 (indicated by the heavy arrow in the image at 03:13 UT). The two ribbons of the second flare straddled regions of opposite polarity (Fig. 2). The third flare was observed at 06:09 and 06:59 UT (see the black arrows in filtergrams of Fig. 4). It was a confined microflare and was accompanied by a small surge.

\section{Filament disturbances and magnetic interaction at the two filament ends}

In Table 1, the temporal relationship between the three flares and the filament activations is summarized. We see that the filament had been disturbed before each flare; this suggested that the flares were directly associated with the filament activations. The first activation is demonstrated by the offband $\mathrm{H}_{\beta}$ filtergrams and the corresponding Dopplergrams in Fig. 3a; and the second and third activation, in Fig. 4. We stress that our Dopplergrams were made by using the subtraction technique at $\pm 0.24 \AA$ from the line center of $\mathrm{H}_{\beta}$. When there is flaring, the spectral line might be in emission; the Dopplershift obtained by subtracting images at the red and blue wings is difficult to interpret. For this reason, the Dopplershifts in flare ribbons have not been taken into account. In Fig. 4, we have chosen the window which only contains the filament. To demonstrate the relation of the filament acti- vations and the magnetic interactions at the two filament ends (Sect. 2), in Fig. 5, we have shown the evolution of photospheric magnetic field by the time sequences of line-of-sight magnetograms with superposition of filament threads.

\section{1. the first activation}

The process of the first activation manifested as follows (Fig. 3a). Firstly, about one hour before the flare occurrence, the first $\mathrm{H}_{\beta}$ observation at 00:16 UT showed that the NE end had apparently stretched out above the $\delta$ spot while the SW end remained rooted; the whole filament showed blue shift. This suggested that the filament was lifted up from this end. Secondly, a discontinuity of velocity with both blue and red shift patches appeared at its top at 00:55 UT, suggesting the filament was twisting and possibly expanding as it lifted up (Schmieder et al. 1985; Kurokawa et al. 1987). Thirdly, at 01:24 UT, while the SW end stayed anchored, the NE end further detached from the $\delta$ spot. The filament became darker and thicker, indicating the presence of either enhanced microturbulence or twisted motions (Schmieder et al. 1991). Finally, by 01:54 UT when the first flare closed to its maximum, the central part of the filament divided into two threads and the NE end became rooted in the $\delta$ spot again (also see Fig. 2).

It is clear that the first activation was mainly manifested by the NE end ascending and detaching from the $\delta$ spot. Beneath this end, the transverse field of the photosphere was strong and sheared, and the major magnetic fluxes of the P1 and F1 tightly squeezed each other, leading to a steep horizontal gradient in the line-of-sight field between them (Fig. 2b and Fig. 5). Across the segment of the magnetic neutral line below the end, marked by the white bar in the frame at 01:52 UT in Fig. 5 (and the black bar in the vector magnetogram in Fig. 2b), the field gradient from positive to negative polarity within a distance of 4 arcsecs was at least $0.12 \mathrm{G} \mathrm{km}^{-1}$. Along this neutral line, the absolute average value of transverse field strength was larger than $1000 \mathrm{G}$, and the average angle difference between the azimuth of the measured transverse field and the neutral line was less than $30^{\circ}$. The magnetic fields at this end are so strong that it becomes difficult to measure shear change and flux evolution. However, as mentioned in Sect. 2, a noticeable characteristic of the two sunspots was the rotation of their axis. Similar to the situation illustrated in Fig. 5 from Rompolt \& Bogdan (1986) and Fig. 5 from Martin (1990), such rotation, considered as anti-parallel motion (shear motion), could lead to the development of magnetic shear in the filament channel (also see Wang \& Shi 1994; Gaizauskas et al. 1994). Moreover, the small change in the strong magnetic field seen here might be enough to influence the topology and stability of the filament magnetic field (see Gaizauskas 1989). Therefore, the first activation is related 


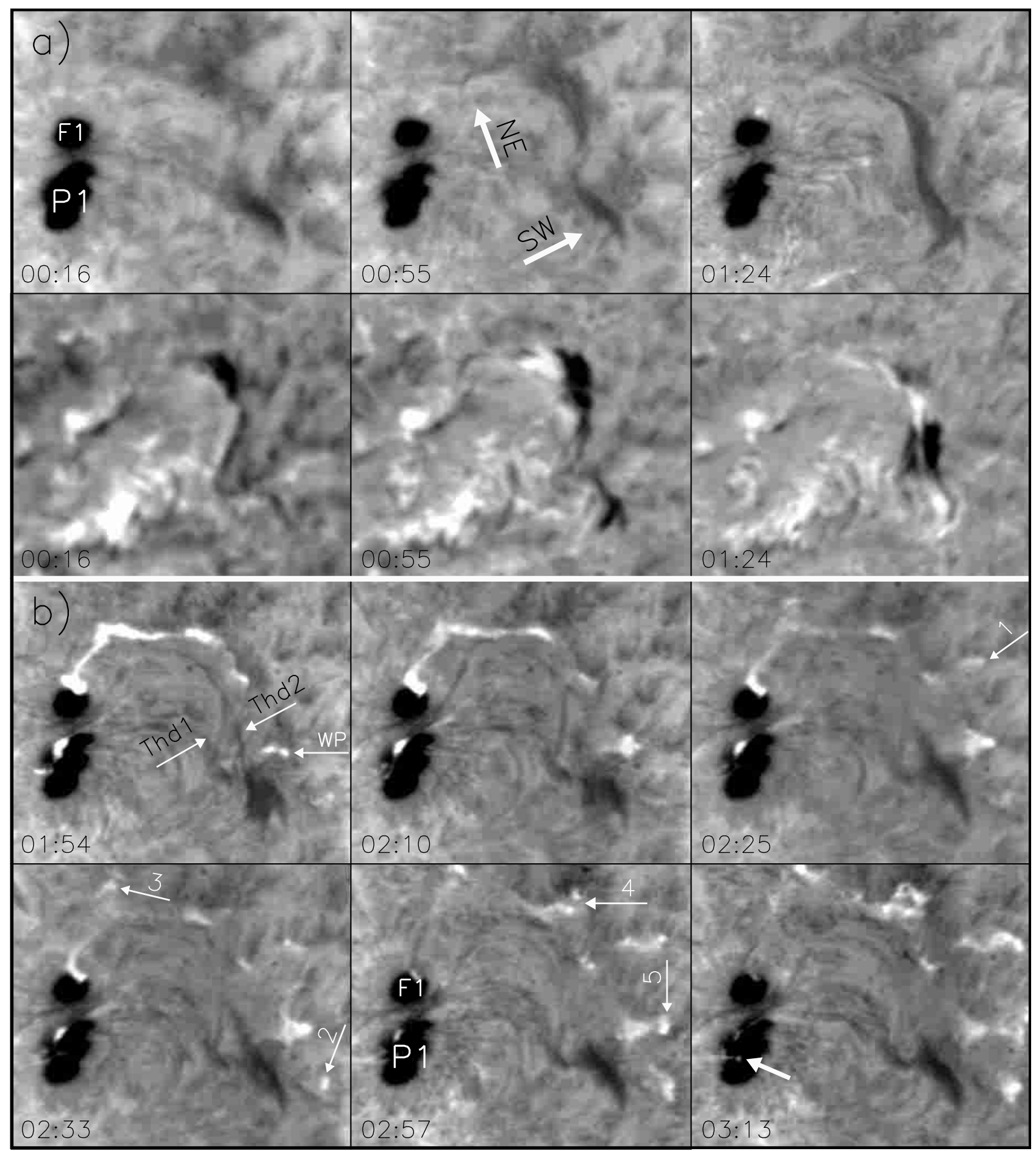

Fig. 3. a) Time sequences of offband $H_{\beta}$ filtergrams (upper) and $H_{\beta}$ Dopplergrams (lower) show the first filament activation. b) Time sequences of offband $\mathrm{H}_{\beta}$ filtergrams show the development of the second flares; the small arrows and letters " 1 ", " 2 ", "3", "4", "5" and "WP", and the heavy arrow indicates the bright patches of the second flare. The FOV is the same as in Fig. 2

to the strong magnetic shear existing at this end, as suggested by the transverse field measurement.

\subsection{The second and third activations}

Before and during the second flare, the filament underwent the second activation (Table 1 and Fig. 4). This activation mainly occurred in the Thd 2 with successive upward and downward motions, while the whole Thd1 showed no obvious change in $\mathrm{H}_{\beta}$ filtergrams and only slow upward motion. The main features of the activation in Thd2 are characterized by following: (a) the northern part of the Thd 2 first moved upwards from 01:54 to 02:00 UT. This led to its detachment from the Thd 1 at the $\mathrm{B}$ point where the Thd1 appeared to be broken (see the arrow in filtergram at 02:10 UT). The Thd2 then descended and connected 


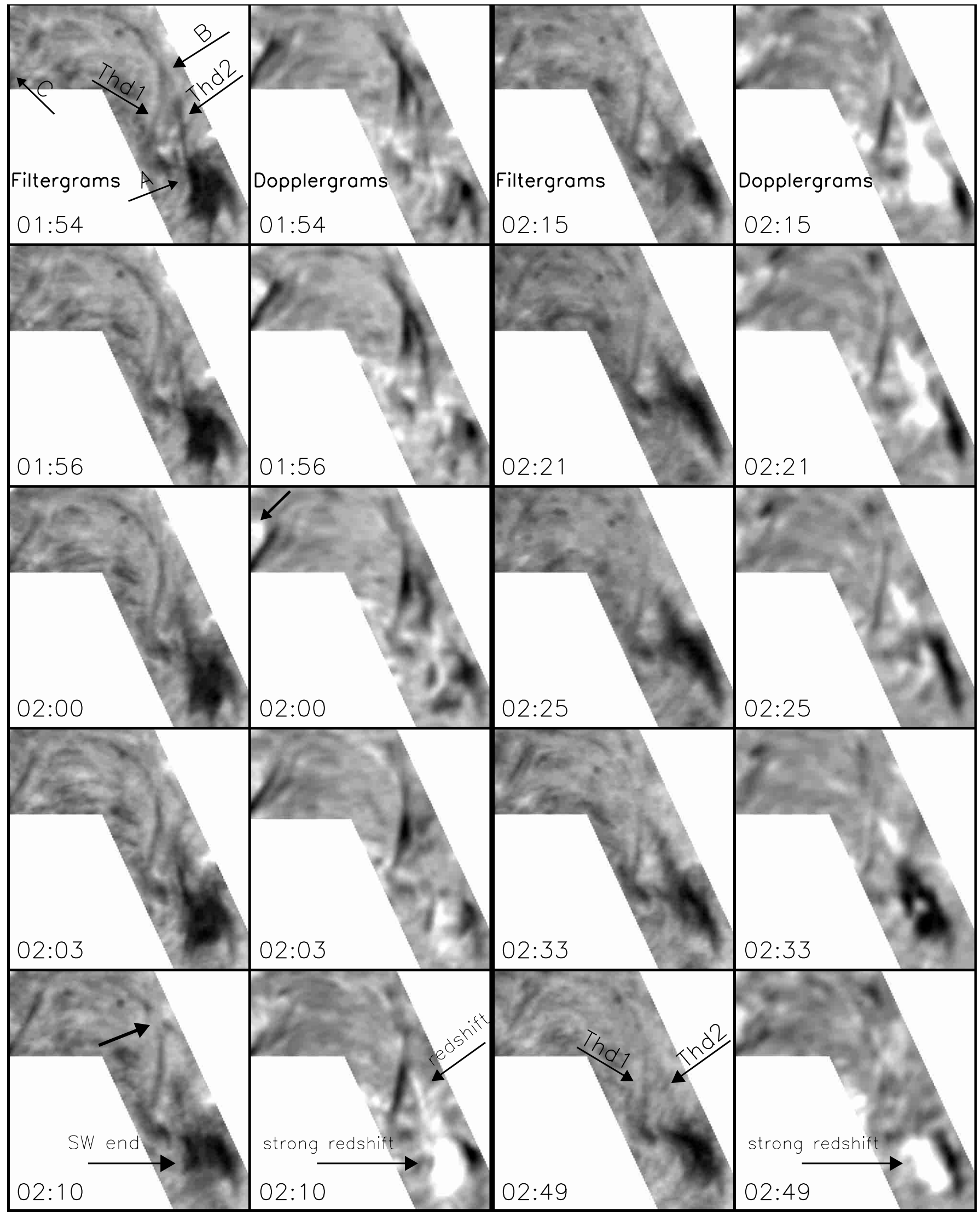

Fig. 4. Time sequences of offband $\mathrm{H}_{\beta}$ filtergrams (the first and third columns) and $\mathrm{H}_{\beta}$ Dopplergrams (the second and fourth columns) show the second and third filament activations. In Dopplergrams, black (white) represents blue (red) shifts. The FOV is $125^{\prime \prime} \times 125^{\prime \prime}$ 


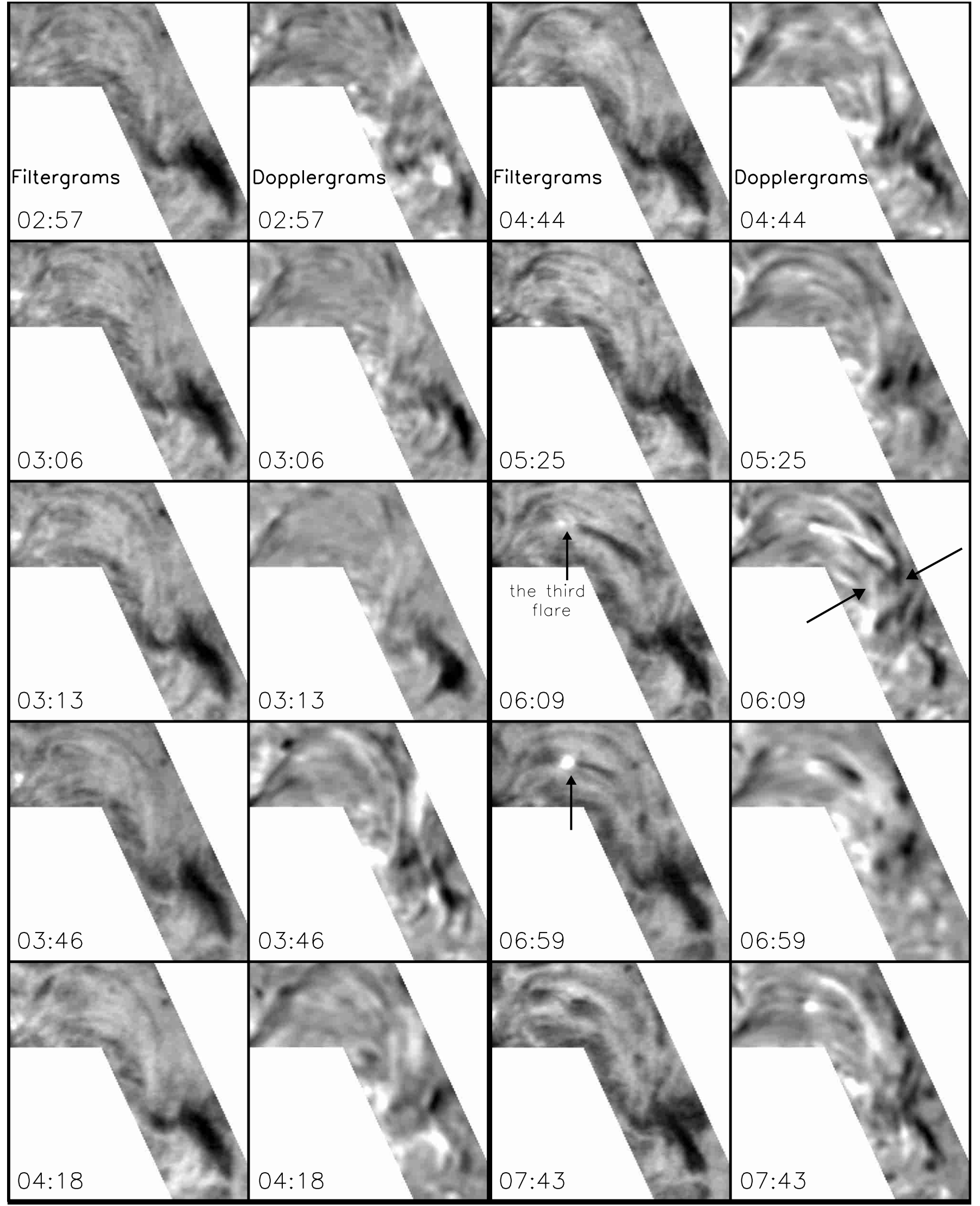

Fig. 4. continued 


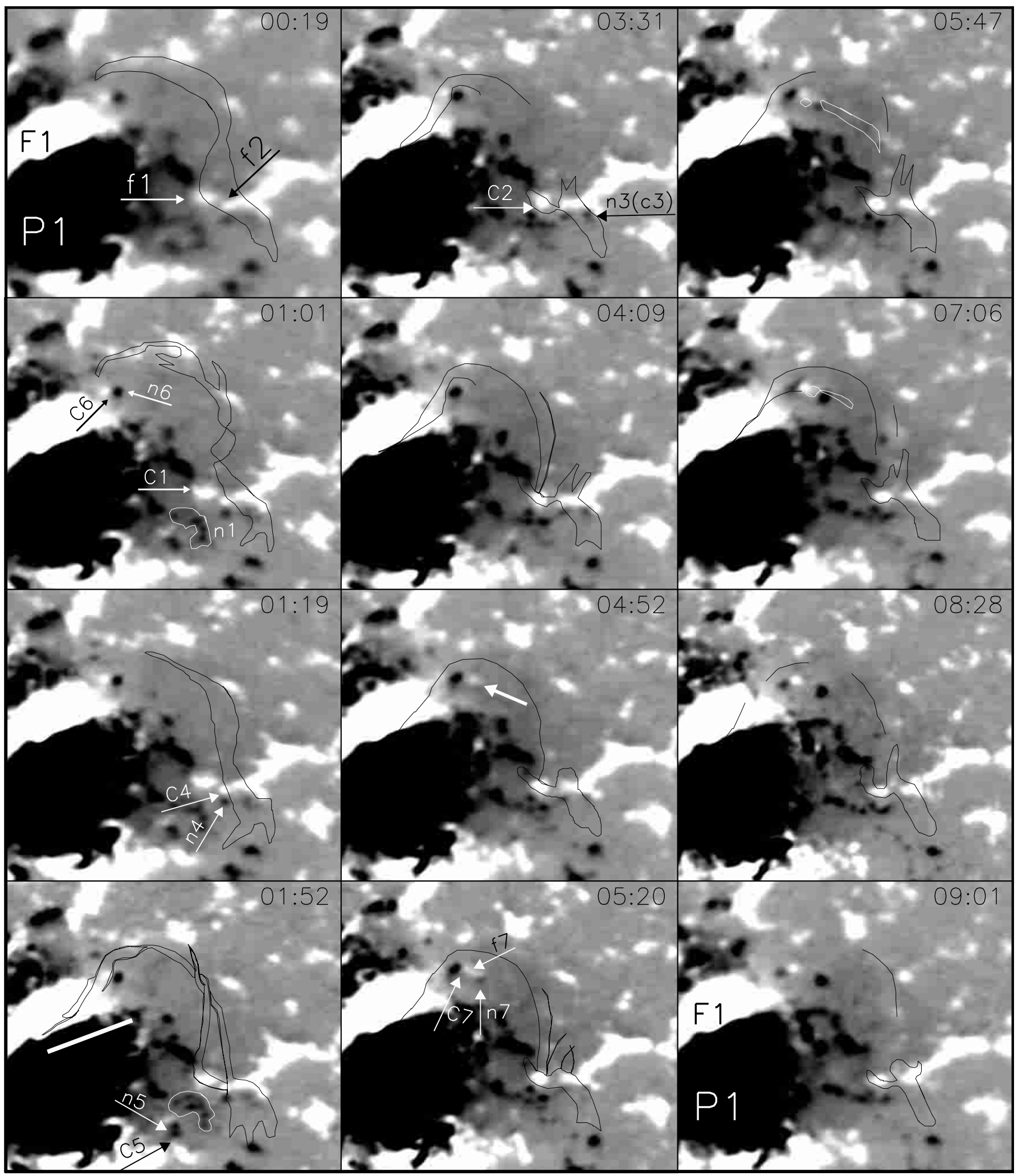

Fig. 5. Time sequences of line-of-sight magnetograms with superposition of filament as dark contours and lines. The magnetograms are displayed in greyscale formats with brighter (darker) patches for positive (negative) polarities. To show the changes in magnetic flux of small scale against the backdrop of a slowly-evolving stronger magnetic field in stable $\delta$ spot, the dynamic range for the display is set up with $\pm 100 \mathrm{G}$. In the figure, f1, f2 and f7 are positive magnetic flux patches, and n1 and n3-7, negative ones. C1-7 mark the CMFs. The FOV is $200^{\prime \prime} \times 175^{\prime \prime}$

again with the Thd1 from 02:10 to 02:49 UT. The change of its motion from blueshift to redshift occurred in the in- terval between 02:00 and 02:10 UT. We note that Thd2 showed neither red nor blue shift at 02:03 UT. Finally, 


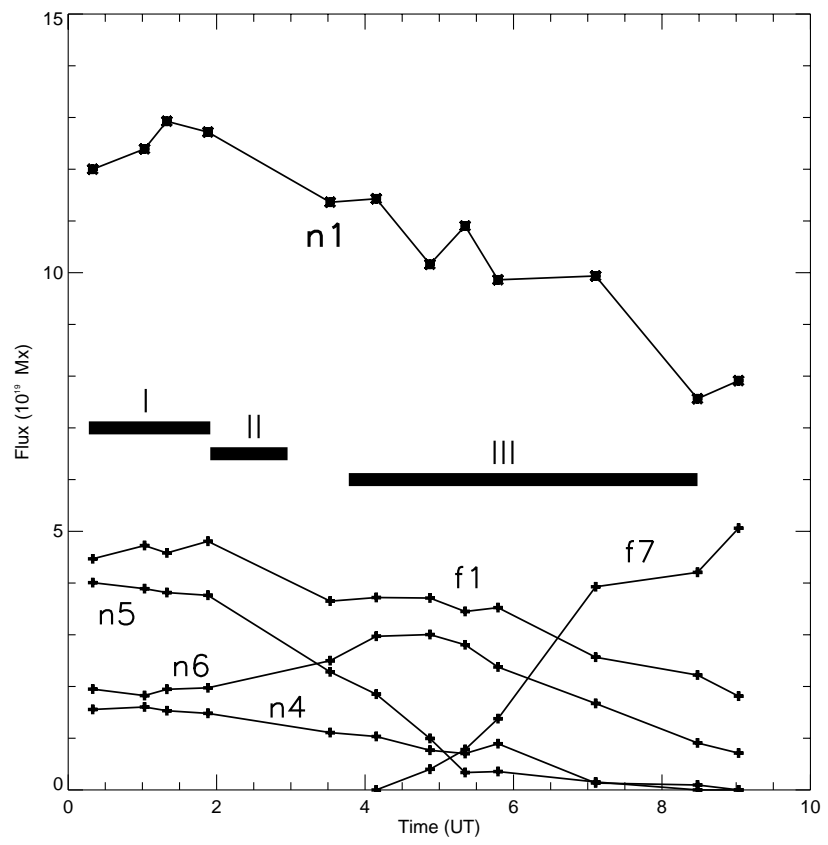

Fig. 6. Flux changes in the negative components of $\mathrm{C} 2$ (the n1), C4 (the n4), C5 (the n5) and C6 and C7 (the n6), in the positive components of $\mathrm{C} 1$ and $\mathrm{C} 2$ (the $\mathrm{f} 1$ ), and in the positive pole of the EFR (the f7). The periods of the three filament activations are shown as horizontal bars

at 02:49 UT, the Thd2 faded. (b) Since the two threads stretched out to the NE end, the BC section was also disturbed and a red shift patch appeared towards its north (see the arrow in Dopplergram at 02:00 UT). (c) More dramatic changes took place at the SW end, which consisted of fine structures. These structures showed successively upward motion followed by strong redshift. Such Dopplershift suggested surging activity in these structures (Schmieder et al. 1994). (d) Between 02:10 and 02:33 UT, a velocity pattern with alternating red and blue shift was formed in the whole filament, indicating that the two threads were twisted (Rust et al. 1994; Wang et al. 1996a; Jiang \& Wang 2000).

Near the two filament ends, several cancelling magnetic features (CMFs) were identified in photospheric magnetograms throughout the entire observation (Fig. 5). Around the SW end, the positive polarity patches, f1 and f2, intruding into the backdrop of the negative polarity flux around the P1, led to obvious cancellation of magnetic flux between f1, f2 and P1. Five CMFs, C1, C2, C3, $\mathrm{C} 4$ and $\mathrm{C} 5$, were identified here. The $\mathrm{C} 1$ was formed by the collision of the f1 with the negative flux on its northeastern side. A negative flux patch within the white contour, n1, converged toward the f1 and closely contacted it, forming the $\mathrm{C} 2$ at 03:31 UT. Cancellation in C2 persisted until the end of observation. The flux cancellation in $\mathrm{C} 1$ and $\mathrm{C} 2$ was manifested by obvious flux loss in $\mathrm{f} 1$. The small C3 and C4 were formed by negative flux patches, $\mathrm{n} 3$ and $\mathrm{n} 4$, and positive flux $\mathrm{f} 2$, respectively; the C5 was formed by the negative patch, n5, approaching to and then cancelling with positive flux at 01:52 UT. The C5 lasted until 05:47 UT when the n5 had completely disappeared. These CMFs seemed to be responsible for the generation of a small surge. Adjacent to the NE end, there also was a CMF, C6, whose negative component was denoted as n6. The red shift motions described above during the second filament activation mainly appeared above these cancellation sites. Similar behavior in disturbed filaments was also noticed by Wang et al. (1996a) and Jiang \& Wang (2000).

After a relatively quiescent period from 03:06 to 03:13 UT, the filament was re-activated at 03:46 UT; red shift patches again appeared inside the SW end and towards the north of the BC section (Fig. 4). It is noticed that the filament consisted of several threads at 05:25 UT. By 06:09 UT, when the microflare and the small surge occurred, two clear bands of red and blue shift appeared in the filament (shown by the arrows). A similar velocity pattern also appeared at 07:43 UT. In the course of the third activation, when the flux cancellation near the SW end continued, an emerging flux region (EFR) appeared in the vicinity of n6 (Fig. 5). The EFR firstly appeared as a small positive patch at 04:52 UT (see the black heavy arrow); the positive and negative poles, $\mathrm{f} 7$ and $\mathrm{n} 7$, showed obvious separating motion, normally associated with an EFR. As a result, the $\mathrm{f} 7$ was driven cancel with the $\mathrm{n} 6$ and thereby formed another CMF, C7. The microflare and surge directly occurred above these emerging and cancelling sites (see the white contour in the frames at 05:47 and 07:06 UT).

To show the possible relationship between filament activations and flux cancellation and emergence near the two filament ends, in Fig. 6, we plot the flux changes in the negative components of $\mathrm{C} 2$ (the $\mathrm{n} 1$ ), $\mathrm{C} 4$ (the n4), C5 (the n5) and $\mathrm{C} 6$ and $\mathrm{C} 7$ (the n6), in the positive components of $\mathrm{C} 1$ and $\mathrm{C} 2$ (the $\mathrm{f} 1$ ), and in the positive pole of the EFR (the f7). The periods of each activation are plotted as black horizontal bars. We see that all of the cancelling components near the SW end gradually lost their flux, suggesting considerable flux cancellation here. Therefore, the appearance of CMFs and continuous flux loss near this end seem to be a condition for the destabilisation of the filament. Note that the flux in the $\mathrm{f} 7$ obviously increased while in the n6, concurrently decreased during the third activation. It is likely that this activation was related to the EFR and its driven flux cancellation.

\section{Conclusion and discussion}

During an eight-hour observation, an active-region filament underwent three successive activations with different characteristics. The first activation had an asymmetrical nature: the SW end was anchored while the other end was lifted up and detached from the $\delta$ spot. The filament went through a typical preflare activation process of rising, expanding and enhanced twisting motion (similar to Schmieder et al. 1985). After this activation, the filament separated into two components, Thd1 and Thd2. In the course of the second activation, only the Thd 2 was 
disturbed while the Thd1 remained nearly stationary. The Thd2 first ascended, then descended and finally disappeared; in the meantime, strongly downward mass motion appeared in the SW end. In this process, the whole filament showed alternating red and blue shifts, implying surging activity with twisted motions. Finally, during the third activation, twisted filament structures and velocity patterns appeared again. By the next day, the filament still consisted of two threads.

The photospheric magnetic field near the two filament ends showed obvious changes during the filament activations. Beneath the NE end, the two sunspots had strong transverse fields which were sheared, and they underwent obvious shear motion. We believe that such photospheric motion could slowly induce magnetic field changes in the filament, and eventually led to its disturbances. Particularly, since the NE end rose during the first activation, following a scenario proposed by Moore et al. (1984; and Moore \& Rabin 1985), we tentatively interpret this activation as follows: the shear motion led to the development of magnetic shear near the NE end; when the shear reached a critical limit, the field started to reconnect, so the NE end lost its connection to the photosphere and the overall field configuration became unstable. This resulted in further reconnection and the $\mathrm{NE}$ end being expelled upward. In such a scenario, the photospheric magnetic reconnection was an essential ingredient. Near the SW end, the collision of opposite polarity magnetic flux led to obvious flux cancellation. As shown by Wang \& Shi (1993) and Wang (1995), flux cancellation is most likely a steady magnetic reconnection between the two cancelling magnetic components with opposite polarity, which was slow and steady in the photosphere and appeared to be an important mechanism to transport the magnetic complexity upward. Therefore, as suggested by Moore \& Roumeliotis (1992), cancellation reconnection could slowly destabilise the filament by changing its linkage to the photosphere and inducing current in it. The EFR and its driven cancellation reconnection near the NE end might play the same role in the third activation. From the above discussion, the photospheric magnetic reconnection, driven by the shear motion, flux cancellation and bipole emergence, seem to be a promising mechanism for activating the filament and driving the surging activity. However, the following questions are open: (a) what is the critical limit of magnetic shear which can lead to reconnection in the photosphere? (b) how much flux loss by flux cancellation is required to effect the stability of filament?

Following these activations, flares with different importance occurred on both side of the filament. The first flare was a two-ribbon flare and its ribbons did not spread but simply faded in situ. One ribbon of the second flare spread out significantly from the filament axis. In the classical two-ribbon flare scenario (Sturrock 1966; Hirayama 1974; Kopp \& Pneuman 1976), the filament eruption led to a complete opening of the overlying coronal field. The flare was produced by closing-back reconnection. However, the filament studied in this paper was continuously activated but did not erupt, suggesting that its disturbance was not violent enough to completely open the overlying magnetic fields. Thus, the flare mechanism we can visualize is only a partial opening of the overlying field. To partially open a coronal field, it is quite possible that the filament magnetic fields had an interaction with the field in the filament environment, which was implied by the filament behaviors in the first and second activations. Similar examples were recently given by McAllister et al. (1996) and Khan et al. (1998).

The appearance of twisted structures and motions is common in disturbed filament, which possibly resulted from the intrinsic nature of the filament (twisted flux tube, see Rust \& Kumar 1994; Low 1995; Tandberg-Hanssen 1995; Aulanier et al. 1998; Schmieder et al. 2000) or from magnetic reconnection between non-twisted structures (Martin \& McAllister 1997; Zirker et al. 1997). In this example, the twisted magnetic field of the filament was suggested by the presence of the two twisted threads and their velocity pattern during the second activation. Based on an ad hoc assumption that before disturbance a filament is tightly twisted, Wang et al. (1996a) identified such velocity pattern as an untwisting rotation, while Rust et al. (1994) interpreted it as a signature of an expanding helix. In the case we report here, it is clear that such a pattern did not originate from the disturbance of the whole filament. Rather, it is due to the activation of the Thd2. As the result of the disturbance, the undisturbed Thd1 remained and the disturbed Thd2 disappeared. Further observation are needed to clarify the origin of twisted motions in disturbed filaments and the ultimate causes of filament instability.

Acknowledgements. The authors thank Yuanyong Deng and Wei Li for useful discussions. We are indebted to the Huairou staff for good observations. The authors are very grateful to the referee B. Schmieder for critical comments and valuable suggestions. This work is supported by the National Natural Science Foundation of China under grants 19791090, 19973009.

\section{References}

Ai, G., \& Hu, Y. 1986, Pub. Beijing Astron. Obs., 8, 1

Aulanier, G., Démoulin, P., van Driel-Gesztelyi, L., et al. 1998, A\&A, 335, 309

Feynman, J., \& Martin, S. F. 1995, JGR, 100(A3), 3355

Gaizauskas, V. 1989, Solar phys., 121, 135

Gaizauskas, V., Harvey, K. L., \& Proulx, M. 1994, ApJ, 422, 883

Hermans, L., \& Martin, S. F. 1986, in CPP NASA Conference, ed. A. Poland, CP 2442, 369

Hirayama, T. 1974, Solar Phys., 34, 323

Jiang, Y, \& Wang, J. 2000, A\&A, 356, 1055

Khan, J. I., Uchida, Y., McAllister, A. H., et al. 1998, A\&A, 336,753

Kopp, R. A., \& Pneuman, G. W. 1976, Solar Phys., 50, 85

Kurokawa, H., Hanaoka, Y., Shibata, K., et al. 1987, Solar Phys., 108, 251

Low, B. C., \& Hundhausen, J. R. 1995, ApJ, 443, 818

Martin, S. F. 1980, Solar Phys., 68, 217 
Martin, S. F. 1990, in IAU Colloq. 117, ed. V. Ruz̆djak, \& E. Tandberg-Hanssen (Springer-Verlag), 1

Martin, S. F., \& Livi, S. H. 1992, in IAU Colloq. 133, ed. Z. Sv̌estka, B. V. Jackson, \& M. E. Machado (SpringerVerlag), 33

Martin, S. F., \& McAllister, A. H. 1997, Proceedings of Chapman Conference on Coronal Mass Ejections, 127

McAllister, A. H., Kurokawa, H., Shibata, K., et al. 1996, Solar Phys., 169, 123

Moore, R. L., Hurford, G. J., Jones, H. P., et al. 1984, ApJ, 276,379

Moore, R. L., \& Rabin, D. 1985, ARA\&A, 23, 239

Moore, R. L., \& Roumeliotis, G. 1992, in IAU Colloq, 133, Eruptive Solar Flares, ed. Z. Š̌estka, B. V. Jackson, \& M. E. Machado (Springer-Verlag, Berlin), 69

Mouradian, Z., Soru-Escaut, I., Hiei, E., et al. 1998, Solar Phys., 180, 313

Raadu, M. A., Schmieder, B., Mein, M., et al. 1988, A\&A, 197, 289

Rompolt, B., \& Bogdan, T. 1986, in CPP NASA Conference, ed. A. Poland, CP 2442, 81

Rompolt, B. 1990, Hvar Obs. Bull., 14(1), 37

Rust, D. M., Nakagawa, Y., \& Neupert, W. M. 1975, Solar Phys., 41, 397

Rust, D. M., \& Kumar, A. 1994, Solar Phys., 155, 69

Rust, D. M., Sakurai, T., Gaizauskas, G., et al. 1994, Solar Phys., 153, 1

Schmieder, B., Raadu, M. A., \& Malherbe, J. M. 1985, A\&A, 142,249
Schmieder, B., Raadu, M. A., Démoulin, P., et al. 1989, A\&A, 213, 402

Schmieder, B. 1990, in IAU Colloq. 17, Dynamics of Quiescent Prominences, ed. V. Ruždjak, \& E. Tandberg-Hanssen (Springer-Verlag), 85

Schmieder, B., Fontenla, J., \& Tandberg-Hanssen, E. 1991, A\&A, 252, 343

Schmieder, B., Golub, L., \& Antiochos, S. K. 1994, ApJ, 425, 326

Schmieder, B., Delannée, C., Deng., Y, et al. 2000, A\&A, in press

Simon, G., van Driel-Gesztelyi, L., Schmieder, B., \& Mein, N. 1986, in CPP NASA Conf., ed. A. Poland, CP 2442, 229

Sturrock, P. A. 1966, Nature 211, 695

Tandberg-Hanssen, E. 1995, The Nature of Solar Prominences (Kluwer Academic Publishers, Dordrecht)

Tang, F. 1986, Solar Phys., 105, 399

Vrs̆nak, B., Ruždjak, V, \& Rompolt, B. 1991, Solar Phys., 136, 151

Wang, J. 1993, Publ. Beijing Astron. Obs., 19, 36

Wang, J., \& Shi, Z. 1993, Solar Phys., 143, 119

Wang, J., \& Shi, Z. 1994, Solar Phys., 155, 285

Wang, J. 1995, Science in China, Series A, 25(2), 198

Wang, J., Shi, Z., \& Martin, S. F. 1996a, A\&A, 316, 201

Wang, J., Shi, Z., Wang, H., et al. 1996b, ApJ, 456, 861

Zirin, H., \& Liggett, M. 1987, Solar Phys., 113, 263

Zirker, J. B., Martin, S. F., Harvey, K., \& Gaizauskas, V. 1997, Solar Phys., 175, 27 